\title{
Evaluation of a pharmacy-driven methicillin-resistant Staphylococcus aureus surveillance protocol in pneumonia
}

\author{
Sarah Dunaway ${ }^{1} \cdot$ Kara W. Orwig $^{1} \cdot$ Zachary Q. Arbogast $^{2} \cdot$ Zachary L. Myers $^{2} \cdot$ James A. Sizemore $^{1,2}$. \\ Stephanie E. Giancola ${ }^{3}$
}

Received: 9 February 2018 / Accepted: 26 April 2018 / Published online: 2 May 2018

๑) Springer International Publishing AG, part of Springer Nature 2018

\begin{abstract}
Background Methicillin-resistant Staphylococcus aureus (MRSA) is an important cause of pneumonia and clinicians must determine when empiric antimicrobial therapy directed toward MRSA is needed. Objective To evaluate the effect of a pharmacy-driven protocol utilizing the nasal swab MRSA polymerase chain reaction (PCR) test to discontinue vancomycin on duration of vancomycin therapy and clinical outcomes in patients with suspected community-acquired pneumonia (CAP) or healthcare-associated pneumonia (HCAP). Setting A teaching hospital in Huntington, WV, USA. Methods This retrospective study included adult patients who received at least one dose of vancomycin for suspected CAP or HCAP. The pre-intervention group consisted of patients prior to the addition of the nasal swab MRSA PCR test to the CAP/HCAP order set. The postintervention group consisted of patients after the addition of the nasal swab MRSA PCR test to the CAP/HCAP order set. Main outcome measure The primary outcome was vancomycin hours of therapy. Results Of the 196 patients included in the study, 121 patients were in the pre-intervention group and 75 patients were in the post-intervention group. The median duration of vancomycin therapy was significantly shorter in the post-intervention group than the pre-intervention group (49 vs. $18 \mathrm{~h}, p<0.001$ ). There were no statistically significant differences in the secondary outcomes including hospital length of stay, 30-day readmission rate, and in-hospital all-cause mortality. Conclusion The addition of a pharmacy-driven protocol utilizing the nasal swab MRSA PCR test was associated with shorter duration of empiric vancomycin therapy by approximately $31 \mathrm{~h}$ per patient without increasing adverse clinical outcomes.
\end{abstract}

Keywords CAP $\cdot$ HCAP · MRSA $\cdot$ Nasal swab · Pneumonia $\cdot$ United States · Vancomycin

\section{Impacts on practice}

- Clinicians are often reluctant to remove antibiotics directed toward MRSA due to the lack of good-quality respiratory cultures.

- Hospital pharmacists may make important clinical decisions based on the nasal swab MRSA PCR test result

Stephanie E. Giancola

Stephanie.e.giancola.ctr@mail.mil

St. Mary's Medical Center, Huntington, WV, USA

2 Marshall University School of Pharmacy, Huntington, WV, USA

3 Department of Pharmacy, Brooke Army Medical Center, 3551 Roger Brooke Dr., Fort Sam Houston, TX 78234, USA without negatively impacting outcomes in patients with pneumonia.

- Pharmacy-driven protocols utilizing the nasal swab MRSA PCR test may reduce anti-MRSA antibiotic exposure.

\section{Introduction}

Pneumonia caused by methicillin-resistant Staphylococcus aureus (MRSA) is associated with significant morbidity and mortality; therefore, initiating empiric antibiotic therapy directed toward MRSA in patients at risk for this pathogen is vital $[1,2]$. Patients at risk for MRSA pneumonia include those with severe community-acquired pneumonia (CAP) with necrotizing or cavitary lesions or admission to the intensive care unit (ICU) and patients with hospital-acquired and ventilator-associated pneumonia (HAP/VAP) who meet 
criteria defined by the 2016 guidelines by the Infectious Diseases Society of America (IDSA) and American Thoracic Society (ATS) [3, 4]. Additionally, although healthcare-associated pneumonia (HCAP) was removed from the most recent HAP/VAP guidelines, it remains unclear which patients presenting from the community will be considered at higher risk for MRSA pneumonia in the anticipated updates to the CAP guidelines; therefore, many clinicians still include anti-MRSA therapy in the treatment of patients with the previously defined HCAP risk factors $[4,5]$. In concordance with these guidelines, many patients with suspected pneumonia are initiated on broad-spectrum empiric antibiotics, including anti-MRSA therapy. Guidelines recommend that the initial empiric regimen be narrowed based on microbiological etiology. However, it is often difficult to obtain a good-quality lower respiratory tract specimen for culture, leaving physicians with poor quality specimens or no culture data at all. For this reason, physicians are often reluctant to remove antibiotics directed toward MRSA.

Recently, the use of nasal MRSA screening for antibiotic de-escalation in pneumonia has sparked interest [6]. The nasal screening tests for MRSA are rapid diagnostic tools with results returning in as little as $90 \mathrm{~min}$. Traditionally, they have been used as an infection prevention tool in order to reduce the transmission of MRSA by identifying patients in which to initiate contact precautions and decolonization regimens [7-9]. Recent studies, however, have identified a potential use of nasal MRSA screening to guide antimicrobial therapy in patients with suspected pneumonia [9-14]. These studies have consistently demonstrated that these tests have a high negative predictive value ( $>95 \%)$ for MRSA pneumonia. Among these studies was a retrospective evaluation of the nasal swab MRSA polymerase chain reaction (PCR) test conducted by our group, which demonstrated a negative predictive value of $98.6 \%$ and a potential to prevent 300 of $782(38.4 \%)$ days of anti-MRSA therapy in all ICU and intermediate care unit patients with suspected pneumonia [14]. Therefore, it was incorporated into the CAP/HCAP order set at St. Mary's Medical Center (SMMC).

Although there has been a substantial amount of recent data demonstrating a high negative predictive value of nasal MRSA screening in suspected pneumonia, there are limited data on clinical outcomes and the impact on duration of therapy when this test is used to discontinue anti-MRSA antibiotics, particularly when used as a pharmacy-driven intervention.

\section{Aim of study}

The objective of this study was to evaluate the effect of a pharmacy-driven protocol utilizing the nasal swab MRSA PCR test to discontinue vancomycin on duration of vancomycin therapy and clinical outcomes in patients with suspected CAP or HCAP.

\section{Ethics approval}

The Marshall University Institutional Review Board approved the study and informed consent was waived (IRBNet ID\# 935525-1).

\section{Methods}

\section{Study design and patient population}

This single-center, quasi-experimental study was conducted at SMMC, a 393-bed teaching hospital in Huntington, WV, USA. All adult inpatients initiated on the CAP/HCAP order set with vancomycin ordered between January 1, 2016 and December 30, 2016 were screened for enrollment. The preintervention group consisted of patients prior to the addition of the nasal swab MRSA PCR test to the CAP/HCAP order set between January 2016 and June 2016. The postintervention group consisted of patients after the addition of the nasal swab MRSA PCR test to the CAP/HCAP order set between July 2016 and December 2016. Additional inclusion criteria included age $\geq 18$ and $\leq 89$ years and nasal swab MRSA PCR test completed within $24 \mathrm{~h}$ of being admitted for the post-intervention group. Patients were excluded if they had a MRSA infection elsewhere (e.g. skin and soft tissue infection, blood) at the time of the nasal swab MRSA PCR result.

\section{Intervention}

Prior to the study period, there was an existing order set that was commonly utilized for patients presenting with suspected CAP or HCAP. According to the order set, vancomycin was ordered with an automatic $72 \mathrm{~h}$ stop date for patients with CAP who were admitted to the ICU or had evidence of cavitary or necrotizing lesions and all patients with HCAP risk factors. Additionally, all intravenous vancomycin orders were automatically dosed by pharmacists according to hospital protocol with a goal trough of $15-20 \mathrm{mcg} / \mathrm{mL}$. Beginning in July 2016, the nasal swab MRSA PCR test was added as an automatic order to the CAP/HCAP order set. During the study period, nasal swab MRSA PCR testing was performed using the Xpert MRSA Assay in the GeneXpert Dx System (Cepheid, Sunnydale, CA). The SMMC chemistry lab processes the nasal swab upon receipt of the specimen and results are typically available within ninety minutes. If the result of this test was negative and there was no evidence of MRSA 
infection elsewhere, the pharmacist was permitted to discontinue the vancomycin order without a direct physician order. All other antimicrobial management decisions were left to the discretion of the primary treating provider or team, which generally consisted of an attending physician with or without trainees (students, residents, and/or fellows); there were no other interventions implemented for antimicrobial management between the pre-intervention and post-intervention periods. Although the updated HAP/ VAP guidelines were published online at the beginning of the post-intervention period, the removal of HCAP from the guideline did not lead to additional updates in the CAP/HCAP order set since it remained unclear how HCAP would be addressed in the anticipated updates to the CAP guidelines.

\section{Data collection and definitions}

Data collected from each patient's electronic medical record included age, sex, comorbidities, ICU admission, pneumonia type, respiratory culture data (within $48 \mathrm{~h}$ of admission, if available), nasal swab MRSA PCR test result, radiographic data, vital signs, pertinent laboratory data, any antibiotic exposure within the past 90 days, vancomycin data (start and stop date/time), and clinical outcomes data (readmission and mortality). Chest X-ray and CT scan results based on radiology reports were categorized as definitive, possible/probable pneumonia, or negative. Signs of infiltration, consolidation, or cavitary lesions without another possible finding were considered definitive, while any of these or airspace disease with another possible finding was considered as possible/ probable pneumonia. A chest X-ray or CT scan without any signs of infiltration, consolidation, cavitary lesions, or airspace disease was considered negative for pneumonia. Symptoms suggestive of pneumonia included the following: temperature $<36$ or $>38{ }^{\circ} \mathrm{C}$, WBC count $<4000$ or $>11,000$ cells $/ \mathrm{mm}^{3}$, respiratory rate $>20$ breaths $/ \mathrm{min}$, oxygen saturation $<90 \%$, increased cough, increased sputum volume, or sputum purulence $[3,5]$. CAP was defined as suspected pneumonia (based on use of the CAP/HCAP order set) within $48 \mathrm{~h}$ of admission without any HCAP risk factors [3]. HCAP was defined as suspected pneumonia within $48 \mathrm{~h}$ of admission plus any of the following risk factors: hospitalization for at least 2 days within the previous 90 days; residence in a nursing home or long-term care facility; received intravenous antibiotic therapy, chemotherapy, or wound care within 30 days; or attended a hemodialysis clinic within 30 days [5]. The nasal swab MRSA PCR test results were reported as either positive or negative. Sputum cultures were considered MRSA positive if MRSA was isolated in any appreciable degree, as quantitative cultures were rarely performed.

\section{Outcome/endpoints}

The primary outcome was vancomycin hours of therapy. Secondary outcomes included in-hospital all-cause mortality, hospital length of stay (LOS), and 30-day readmission rate for any reason. Hours of therapy were determined by subtracting the start date/time from the stop date/time of the vancomycin order. LOS was rounded to the nearest whole day and was determined by counting each day from the admission date through the discharge date.

\section{Statistical analysis}

We hypothesized that the utilization of the nasal swab MRSA PCR test would decrease the duration of vancomycin therapy by approximately $24 \mathrm{~h}$. Assuming the baseline duration of therapy was $72 \pm 36 \mathrm{~h}$ (based on the $72 \mathrm{~h}$ automatic stop date per the CAP/HCAP order set), a sample size of 47 patients in each group was needed to detect a difference of $24 \mathrm{~h}$ with $90 \%$ power. Categorical data were compared using the Chi squared or Fisher's exact test where appropriate. Continuous data were compared using the Wilcoxon rank sum test since the data are nonparametric. Baseline characteristics that were different between the pre-intervention and post-intervention groups with $p<0.1$ as well as study group were entered into a multivariate analysis that was performed by multiple linear regression to identify independent predictors of hours of therapy. Multiple linear regression was performed by taking the natural log of hours of therapy in order to approximate normal distribution. All tests were twosided with statistical significance set at a $p$ value of $<0.05$. Statistical analyses were performed using Stata 13.0 (College Station, TX).

\section{Results}

A total of 301 patients were screened during the study period. A total of 34 patients were excluded due to a MRSA infection elsewhere, 28 due to age $>89$, and 43 were excluded in the post-intervention group because a nasal swab MRSA PCR test was not obtained within $24 \mathrm{~h}$ of admission. Of the remaining 196 patients who met inclusion criteria, 121 patients were in the pre-intervention group and 75 patients were in the post-intervention group. Patient baseline characteristics are provided in Table 1 . There were significantly fewer males (58.7 vs. $44.0 \%, p=0.04)$ and fewer patients with chronic kidney disease (38.0 vs. $24.0 \%, p=0.04$ ) in the post-intervention group than the pre-intervention group. The post-intervention group also had more patients with heart failure (8.4 
Table 1 Baseline characteristics

\begin{tabular}{|c|c|c|c|}
\hline Characteristic & $\begin{array}{l}\text { Pre-intervention } \\
\text { group }(n=121)\end{array}$ & $\begin{array}{l}\text { Post-intervention } \\
\text { group }(\mathrm{n}=75)\end{array}$ & $p$ value \\
\hline Age, median (IQR) & $71(62-81)$ & $68(55-79)$ & 0.06 \\
\hline Male, n (\%) & $71(58.7)$ & $33(44.0)$ & 0.04 \\
\hline COPD, n (\%) & $52(43.0)$ & $32(42.7)$ & 0.97 \\
\hline Type 2 diabetes, n (\%) & $47(38.8)$ & $24(32.0)$ & 0.33 \\
\hline CKD/ESRD, n (\%) & $46(38.0)$ & $18(24.0)$ & 0.04 \\
\hline Heart Failure, n (\%) & $6(8.4)$ & $17(23.9)$ & 0.01 \\
\hline Chemotherapy, n (\%) & $15(12.4)$ & $14(18.7)$ & 0.23 \\
\hline Immunosuppression $^{\mathrm{a}}, \mathrm{n}(\%)$ & $9(7.4)$ & $2(2.7)$ & 0.21 \\
\hline Injection drug use, n (\%) & $5(4.1)$ & $3(4.0)$ & 1.0 \\
\hline Antibiotic within 90 days, n (\%) & $56(46.3)$ & $40(53.3)$ & 0.34 \\
\hline HCAP, n (\%) & $83(68.6)$ & $64(85.3)$ & 0.01 \\
\hline ICU admission, $\mathrm{n}(\%)$ & $19(15.7)$ & $11(14.7)$ & 0.84 \\
\hline Chest X-ray evidence of pneumonia ${ }^{\mathrm{b}}, \mathrm{n}(\%)$ & & & 0.35 \\
\hline Negative & $8(6.7)$ & $9(12.0)$ & \\
\hline Possible/probable & $87(72.5)$ & $54(72.0)$ & \\
\hline Definitive & $25(20.8)$ & $12(16.0)$ & \\
\hline Oxygen saturation $<90 \%, \mathrm{n}(\%)$ & $12(17.4)$ & $9(12.0)$ & 0.31 \\
\hline Cough, n (\%) & $52(43.0)$ & $50(66.7)$ & $<0.01$ \\
\hline $\mathrm{WBC}>11,000$ cells $/ \mathrm{mm}^{3}, \mathrm{n}(\%)$ & $59(48.8)$ & $35(46.7)$ & 0.78 \\
\hline $\mathrm{WBC}<4000$ cells $/ \mathrm{mm}^{3}, \mathrm{n}(\%)$ & $3(2.5)$ & $5(6.7)$ & 0.26 \\
\hline Sputum production, $\mathrm{n}(\%)$ & $27(22.5)$ & $13(17.3)$ & 0.38 \\
\hline $\mathrm{RR}>20$ breaths/min, $\mathrm{n}(\%)$ & $16(13.2)$ & $11(14.7)$ & 0.78 \\
\hline Temperature $>38^{\circ} \mathrm{C}, \mathrm{n}(\%)$ & $14(11.6)$ & $15(20.0)$ & 0.11 \\
\hline
\end{tabular}

${ }^{a}$ Immunosuppression was defined as active chemotherapy or chronic steroid use

${ }^{\mathrm{b}}$ Chest X-ray evidence categories were defined as follows: signs of infiltration, consolidation, or cavitary lesions without another possible finding was considered definitive; signs of infiltration, consolidation, cavitary lesions, or airspace disease with another possible finding was considered as possible/probable pneumonia; no signs of infiltration, consolidation, cavitary lesions, or airspace disease was considered negative for pneumonia

$I Q R$ interquartile range; $C O P D$ chronic obstructive pulmonary disease; $C K D / E S R D$ chronic kidney disease/end-stage renal disease; $H C A P$ healthcare-associated pneumonia; $W B C$ white blood cell; $R R$ respiratory rate

vs. $23.9 \%, p=0.01)$, more patients with HCAP (68.6 vs. $85.3 \%, p=0.01)$, and more patients with a cough (43.0 vs. $66.7 \%, p<0.01)$ than the pre-intervention group.

In the post-intervention group, the nasal swab MRSA PCR test was positive in 8 patients and negative in 67 patients. Sputum cultures were obtained in 90 patients (53 pre-intervention and 37 post-intervention) and were positive for MRSA in 5 patients ( 4 pre-intervention and 1 post-intervention; $p=0.65)$. Other pathogens isolated included: Pseudomonas aeruginosa $(\mathrm{n}=5)$, Escherichia coli $(\mathrm{n}=3)$, Klebsiella pneumoniae. $(\mathrm{n}=2)$, Streptococcus pneumoniae $(\mathrm{n}=2)$, Haemophilus influenzae $(\mathrm{n}=2)$, Moraxella catarrhalis $(\mathrm{n}=1)$, Serratia marcescens $(\mathrm{n}=1)$, Stenotrophomonas maltophilia $(\mathrm{n}=1)$, and Streptococcus agalactiae $(\mathrm{n}=1)$. The majority of sputum cultures resulted in normal respiratory flora $(n=64)$.
The median duration of vancomycin therapy was $49 \mathrm{~h}$ (interquartile range $35-63 \mathrm{~h}$ ) in the pre-intervention group and $18 \mathrm{~h}$ (interquartile range $13-25 \mathrm{~h}$ ) in the post-intervention group $(p<0.01)$. This means that the duration of empiric vancomycin therapy was shortened by approximately $31 \mathrm{~h}$ per patient. There were no statistically significant differences in the secondary outcomes including hospital LOS, 30-day readmission rate, and in-hospital all-cause mortality as shown in Table 2. In the multiple linear regression analysis (Table 3), the post-intervention group and age were predictors for hours of therapy. In order to interpret the values in Table 3, the inverse natural log must be completed. For example, the inverse natural $\log$ of -0.88 (the coefficient for the post-intervention group) is 0.41 , indicating that the postintervention group is associated with approximately a 59\% reduction in vancomycin hours of therapy. Additionally, the 
Table 2 Secondary outcomes

\begin{tabular}{lccc}
\hline Characteristic & $\begin{array}{l}\text { Pre-intervention group } \\
(\mathrm{n}=121)\end{array}$ & $\begin{array}{l}\text { Post-intervention group } \\
(\mathrm{n}=75)\end{array}$ & $p$ value \\
\hline Length of stay (days), median (IQR) & $6(4-9)$ & $5(3-8)$ & 0.20 \\
30-day readmission, n (\%) & $30(24.8)$ & $16(21.3)$ & 0.58 \\
In-hospital, all-cause mortality, n (\%) & $14(11.6)$ & $5(6.7)$ & 0.26 \\
\hline
\end{tabular}

$I Q R$ interquartile range

Table 3 Multiple linear regression analysis for independent predictors of the natural log of hours of therapy

\begin{tabular}{lllr}
\hline Variable & $\begin{array}{l}\text { Regression } \\
\text { coefficient }\end{array}$ & 95\% CI & $p$ value \\
\hline Post-intervention group & -0.88 & -1.18 to -0.57 & $<0.01$ \\
Age & -0.01 & -0.02 to -0.0003 & 0.04 \\
Gender & -0.03 & -0.31 to 0.24 & 0.81 \\
CKD/ESRD & -0.05 & -0.34 to 0.25 & 0.78 \\
Heart failure & -0.06 & -0.42 to 0.30 & 0.74 \\
HCAP & -0.003 & -0.33 to 0.33 & 0.99 \\
Cough & -0.19 & -0.46 to 0.09 & 0.12 \\
\hline
\end{tabular}

$C I$ confidence interval; $C K D / E S R D$ chronic kidney disease/end-stage renal disease, $H C A P$ healthcare-associated pneumonia

inverse natural log of -0.01 (the coefficient for age) is 0.99 , indicating that the vancomycin hours of therapy is reduced by approximately $1 \%$ for each one year increase in age.

\section{Discussion}

Although MRSA is an uncommon cause of CAP and HCAP [15-17], empiric antibiotic regimens often include coverage for MRSA because when it is the causative pathogen, it is often associated with severe pneumonia leading to significant morbidity and mortality when not treated promptly [3, $5,16]$. However, the overuse of anti-MRSA antibiotics is concerning due to increased resistance, nephrotoxicity, and drug costs, and it is recommended to de-escalate empiric regimens based on culture results $[18,19]$. Since culture data are often lacking for patients with pneumonia, there is a need for alternative methods for determining or ruling out the microbiological etiology. One such method that has gained attention in recent years is the use of nasal MRSA screening. Our study supports the use of the nasal swab MRSA PCR test as a guide for discontinuing anti-MRSA therapy in patients with pneumonia. Implementation of a pharmacydriven protocol to discontinue vancomycin in the setting of a negative nasal swab MRSA PCR test in patients with CAP or HCAP was associated with fewer hours of vancomycin therapy without negatively impacting hospital length of stay, 30-day readmission rate for any reason, or in-hospital allcause mortality rate. Although not assessed in our study, it is possible that this reduction in vancomycin exposure may be associated with a decreased incidence of adverse events (such as nephrotoxicity) and slower emergence of resistance. Additionally, it is reasonable to postulate that the protocol is cost-effective; the reduction of vancomycin use, assumed fewer vancomycin serum levels obtained, and reduction in nursing medication administration time each represent cost savings that would likely offset the cost of the nasal swab MRSA PCR test for each patient.

To our knowledge, only two previous studies assessed clinical outcomes when the nasal swab MRSA PCR test was used to guide discontinuation of anti-MRSA therapy (vancomycin or linezolid) [20, 21]. Baby and colleagues demonstrated a decrease in average anti-MRSA days of therapy per patient from 4.0 to $2.1(p<0.0001)$ and hours of therapy per patient from 74 to $27.4(p<0.0001)$ [20]. There were also no differences in clinical outcomes including length of stay or mortality, but they did demonstrate a reduction in acute kidney injury ( 26 vs. $3.3 \% ; p=0.02$ ). This study was limited by a small sample size ( 30 patients in the PCR group and 27 patients in the pre-PCR group). Willis and colleagues similarly demonstrated a decrease in median vancomycin days of therapy of 2.1 days per patient (4.2 vs. 2.1 days/patient; $p<0.0001$ ) with no differences in in-hospital mortality or length of stay [21]. While the study by Willis and colleagues included a larger sample size (150 patients in each group), $41 \%$ of patients had an acute exacerbation of COPD rather than pneumonia. Antibiotic recommendations for patients with acute exacerbations of COPD do not include specific MRSA-targeted therapy; therefore, the results of that study may be difficult to apply to institutions in which vancomycin is not routinely ordered for that indication [22]. While the results from these studies are similar to those found in our study, there are key differences in the protocols. Both the study by Baby et al. [20] and the study by Willis et al. [21] included a protocol that only allowed pharmacists to order the MRSA PCR test with recommendations given to providers for discontinuation of vancomycin or linezolid; the final decision was ultimately at the discretion of the primary treating provider. Our study included a larger sample size of patients with suspected pneumonia and allowed pharmacists to make the decision to discontinue vancomycin without a direct physician order. Therefore, our study showed that pharmacists could make this clinical decision based on 
the nasal swab MRSA PCR test result without negatively impacting patient outcomes.

Limitations of this study include the single-center, retrospective, quasi-experimental design, which may limit the generalizability of our results. Our study did not involve random assignment and we relied on documentation in the electronic medical record to obtain baseline characteristics. Furthermore, the post-intervention period began immediately after implementation of the intervention; this led to 43 patients excluded in the post-intervention group because the nasal swab MRSA PCR test was not obtained within $24 \mathrm{~h}$, which prompted efforts to educate the nursing staff on the importance of obtaining samples for this test in a timely fashion. If a later time period had been studied for the post-intervention group, it is possible that more potentially eligible patients may have been included and personnel may have become more comfortable with the new protocol, potentially leading to further decreases in duration of therapy. There were several differences in baseline demographics; however, these differences did not substantially impact the primary outcome, as only study group and age were associated with hours of therapy as demonstrated by the multiple linear regression analysis. Inclusion in the study was based on use of the CAP/HCAP order set whether or not the patient had clinical signs and symptoms of pneumonia; however, most patients had at least possible radiographic evidence of pneumonia (90.1\%) and most patients had at least one sign or symptom of pneumonia (84.2\%). Additionally, this is a real-world representation of the utility of the nasal swab MRSA PCR test, as many patients in which pneumonia may ultimately be ruled out are started on broadspectrum antimicrobials and continued on the initial regimen for several days prior to establishing the final diagnosis. Since we did not include patients with HAP or VAP, our results may not be applicable to that patient population. The decision to include the test in our CAP/HCAP protocols was due to the predominance of CAP and HCAP in our previous study (96.5\%). Lastly, the rate of MRSA pneumonia in our study was low at $2.5 \%$; therefore, the results of our study may not be generalizable to facilities with higher rates of MRSA pneumonia.

In addition to these limitations, it should be noted that vancomycin may not have been indicated in many patients who received vancomycin based on the presence of HCAP criteria. Since the introduction of "HCAP" in 2005, increasing evidence suggests that the original HCAP risk factors are not appropriate for identifying patients at risk for multidrugresistant (MDR) pathogens [17]. Instead, risk stratification scores have been developed to identify patients at risk for MRSA and other MDR pathogens who may require broadspectrum empiric therapy including anti-MRSA antibiotics [23, 24]. Updated CAP guidelines are currently in development and are expected to clarify patients at risk for MRSA and other MDR pathogens. Therefore, further evaluation of the impact of the nasal swab MRSA PCR test in combination with a risk stratification score or risk factors identified in the anticipated guidelines is needed.

\section{Conclusion}

The results of this study provide additional evidence that the nasal swab MRSA PCR test may be a useful antimicrobial stewardship tool to guide discontinuation of empiric antiMRSA therapy in patients with suspected CAP or HCAP, particularly when adequate lower respiratory tract cultures are not available. The addition of a pharmacy-driven protocol utilizing the nasal swab MRSA PCR test was associated with a shorter duration of empiric vancomycin therapy by approximately $31 \mathrm{~h}$ per patient without increasing adverse clinical outcomes. Further studies are needed to confirm these results.

Acknowledgements We thank Dr. Todd Gress for his assistance with statistical analysis. This project was supported by the Marshall University School of Medicine Appalachian Clinical and Translational Science Institute (ACTSI). The content is solely the responsibility of the authors and does not necessarily represent the official views of the ACTSI.

Funding This research did not receive any specific grant from funding agencies in the public, commercial, or not-for-profit sectors.

Conflicts of interest The authors declare that they have no conflict of interests.

\section{References}

1. Defres S, Marwick C, Nathwani D. MRSA as a cause of lung infection including airway infection, community-acquired pneumonia and hospital-acquired pneumonia. Eur Respir J. 2009;34(6):1470-6.

2. Rubinstein E, Kollef MH, Nathwani D. Pneumonia caused by methicillin-resistant Staphylococcus aureus. Clin Infect Dis. 2008;46(Suppl 5):S378-85.

3. Mandell LA, Wunderink RG, Anzueto A, Bartlett JG, Campbell FD, Dean NC, et al. Infectious Diseases Society of America/ American Thoracic Society consensus guidelines on the management of community-acquired pneumonia in adults. Clin Infect Dis. 2007;44(Suppl 2):S27-72.

4. Kalil AC, Metersky ML, Klompas M, Muscedere J, Sweeney DA, Palmer LB, et al. Management of adults with hospital-acquired and ventilator-associated pneumonia: 2016 clinical practice guidelines by the Infectious Diseases Society of America and the American Thoracic Society. Clin Infect Dis. 2016. https://doi. org/10.1093/cid/ciw353.

5. American Thoracic Society, Infectious Diseases Society of America. Guidelines for the management of adults with hospitalacquired, ventilator-associated, and healthcare-associated pneumonia. Am J Respir Crit Care Med. 2005;171(4):388-416. 
6. Smith EA, Gold HS, Mahoney MV, Hirsch EB, Giancola SE, Snyder GM, et al. Nasal methicillin-resistant Staphylococcus aureus screening in patients with pneumonia: a powerful antimicrobial stewardship tool. Am J Infect Control. 2017. https://doi. org/10.1016/j.ajic.2017.06.032.

7. Huang SS, Yokoe DS, Hinrichsen VL, Spurchise LS, Datta R, Miroshnik I, et al. Impact of routine intensive care unit surveillance cultures and resultant barrier precautions on hospital-wide methicillin-resistant Staphylococcus aureus bacteremia. Clin Infect Dis. 2006;43(8):971-8.

8. Huang SS, Septimus E, Kleinman K, Moody J, Hickok J, Avery TR, et al. Targeted versus universal decolonization to prevent ICU infection. N Engl J Med. 2013;368(24):2255-65.

9. Chan JD, Dellit TH, Choudhuri JA, McNamara E, Melius EJ, Evans HL, et al. Active surveillance cultures of methicillin-resistant Staphylococcus aureus as a tool to predict methicillin-resistant $S$. aureus ventilator-associated pneumonia. Crit Care Med. 2012;40(5):1437-42.

10. Dangerfield B, Chung A, Webb B, Seville MT. Predictive value of methicillin-resistant Staphylococcus aureus (MRSA) nasal swab PCR assay for MRSA pneumonia. Antimicrob Agents Chemother. 2014;58(2):859-64.

11. Tilahun B, Faust AC, McCorstin P, Ortegon A. Nasal colonization and lower respiratory tract infections with methicillin-resistant Staphylococcus aureus. Am J Crit Care. 2015;24(1):8-12.

12. Robicsek A, Suseno M, Beaumont JL, Thomson RB Jr, Peterson LR. Prediction of methicillin-resistant Staphylococcus aureus involvement in disease sites by concomitant nasal sampling. J Clin Microbiol. 2008;46(2):588-92.

13. Johnson JA, Wright ME, Sheperd LA, Musher DM, Dang BN. Nasal methicillin-resistant Staphylococcus aureus polymerase chain reaction: a potential use in guiding antibiotic therapy for pneumonia. Perm J. 2015;19(1):34-6.

14. Giancola SE, Nguyen AT, Le B, Ahmed O, Higgins C, Sizemore JA, et al. Clinical utility of a nasal swab methicillin-resistant Staphylococcus aureus polymerase chain reaction test in intensive and intermediate care unit patients with pneumonia. Diagn Microbiol Infect Dis. 2016;86(3):307-10.

15. Moran GJ, Krishnadasan A, Gorwitz RJ, Fosheim GE, Albrecht V, Limbago B, et al. Prevalence of methicillin-resistant
Staphylococcus aureus as an etiology of community-acquired pneumonia. Clin Infect Dis. 2012;54(8):1126-33.

16. Self WH, Wunderink RG, Williams DJ, Zhu Y, Anderson EJ, Balk RA, et al. Staphylococcus aureus community-acquired pneumonia: prevalence, clinical characteristics, and outcomes. Clin Infect Dis. 2016;63(3):300-9.

17. Chalmers JD, Rother C, Salih W, Ewig S. Healthcare-associated pneumonia does not accurately identify potentially resistant pathogens: a systematic review and meta-analysis. Clin Infect Dis. 2014;58(3):330-9.

18. Sujatha S, Praharaj J. Glycopeptide resistance in grampositive cocci: a review. Interdiscip Perspect Infect Dis. 2012;2012:781679.

19. O'Driscoll T, Crank CW. Vancomycin-resistant enterococcal infections: epidemiology, clinical manifestations, and optimal management. Infect Drug Resist. 2015;8:217-30.

20. Baby N, Faust AC, Smith T, Sheperd LA, Knoll L, Goodman EL. Nasal methicillin-resistant Staphylococcus aureus (MRSA) PCR testing reduces the duration of MRSA-targeted therapy in patients with suspected MRSA pneumonia. Antimicrob Agents Chemother. 2017. https://doi.org/10.1128/AAC.02432-16.

21. Willis C, Allen B, Tucker C, Rottman K, Epps K. Impact of a pharmacist-driven methicillin-resistant Staphylococcus aureus surveillance protocol. Am J Health-Syst Pharm. 2017;74(21):1765-72.

22. Vogelmeier CF, Criner GJ, Martinez FJ, Anzueto A, Barnes PJ, Bourbeau J. Global strategy for the diagnosis, management, and prevention of chronic obstructive lung disease 2017 report. GOLD executive summary. Am J Respir Crit Care Med. 2017;95(5):557-82.

23. Park SC, Kim EY, Kang YA, Park MS, Kim YS, Kim SK, et al. Validation of a scoring tool to predict drug-resistant pathogens in hospitalized pneumonia patients. Int J Tuberc Lung Dis. 2013;17(5):704-9.

24. Shorr AF, Zilberberg MD, Reichley R, Kan J, Hoban A, Hoffman $\mathrm{J}$, et al. Validation of a clinical score for assessing risk of resistant pathogens in patients with pneumonia presenting to the emergency department. Clin Infect Dis. 2012;54(2):193-8. 